\title{
Usefulness, feasibility and face validity of the interRAI Palliative Care instrument according to care professionals in nursing homes: A qualitative study
}

\section{ABSTRACT}

Background: Nursing homes are important locations for palliative care. High quality palliative care requires an evaluation of the different care needs of the nursing home residents. The interRAI Palliative Care instrument is a comprehensive assessment that evaluates the needs and preferences of adults receiving palliative care.

Objectives: This study aims to evaluate the usefulness, feasibility and face validity of the interRAI Palliative Care instrument.

Design: A qualitative study was conducted, based on the abductive reasoning approach.

Setting: Fifteen nursing homes in Flanders (Belgium).

Participants: Calls for participation were sent out by four umbrella organizations of Flemish nursing homes (Belgium) and at a national conference for nursing home staff. Nineteen care professionals (nurses, certified nursing assistants, psychologists, physiotherapists, quality coordinators and directors) of 15 nursing homes voluntarily agreed to participate in the study.

Methods: Care professionals filled out the interRAI Palliative Care instrument during one year for all nursing home residents with palliative care needs. Data on the usefulness, feasibility and face validity of the interRAI Palliative Care instrument were derived from notes, semi-structured interviews and focus groups with participating care professionals and were thematically analyzed and synthesized. Data were gathered between December 2013 and March 2015.

Results: The interRAI Palliative Care instrument was evaluated as a useful instrument by care professionals in nursing homes. However, it is not always feasible to complete the instrument because of organizational reasons (high workload, lack of computers, lack of integration in the organization, etc.). Furthermore, the face validity of the interRAI Palliative Care instrument could be improved as certain items were evaluated as incomplete, missing, redundant or too complex.

Conclusions: Findings highlight the importance of adapting the content of the interRAI Palliative Care instrument for use in nursing homes. Furthermore, the use of the instrument should be integrated in the organization of daily care routines in the nursing homes. Tackling the critical remarks of care professionals will help to optimize the interRAI Palliative Care instrument and hence support palliative care of high quality in nursing homes.

Key words: Comprehensive geriatric assessment; face validity; feasibility; usefulness; interRAI Palliative Care instrument; Nursing homes; Older adults; Palliative care; Qualitative Research. 


\section{INTRODUCTION}

Palliative care has been defined by the World Health Organization (2002) as "an approach that improves the quality of life of patients and their families facing the problems associated with life-threatening illness, through the prevention and relief of suffering by means of early identification and impeccable assessment and treatment of pain and other problems, physical, psychosocial and spiritual".

In western countries, the number of older people dying in nursing homes is increasing (Davies and Higginson, 2002; Percival and Johnson, 2013; Houttekier et al., 2011; Christopher, 2000). Nursing homes therefore become essential places for the provision of palliative care (Froggatt and Payne, 2006). Palliative care in nursing homes is a complex process, which is replete with many challenges. Examples of challenges include: a lack of available time for dying residents, a lack of knowledge about palliative care, expectations of the family concerning the residents' care, challenges in communicating with other healthcare providers, poor transitions between healthcare settings (home, nursing home, hospital). (Unwin et al., 2010; Wowchuck et al., 2007; Phillips et al. 2006). Furthermore, continuous reassessments of physical, spiritual and psychosocial needs of the residents receiving palliative care are required, especially for people with dementia (Unwin et al., 2010; Kales et al., 2015).

In order to meet some of these challenges and to support the assessment and care planning process, evidence based studies have identified the benefits of using comprehensive geriatric assessments (Scanlan, 2005; Wieland and Hirth, 2003; Mcllfatrick, 2007). A comprehensive geriatric assessment is 'a multidimensional, interdisciplinary diagnostic process to determine the medical, psychological, and functional capabilities of a frail elderly people in order to develop a coordinated and integrated plan for treatment and long-term follow-up' (Rubenstein, 1995; Osterweil et al., 2000; Bernabei, et al., 2000). Based on the findings of a systematic review, the interRAI Palliative Care instrument (interRAI PC instrument) is considered to be the most comprehensive geriatric assessment that has been validated for nursing home residents with palliative care needs (Hermans et al., 2014). It evaluates the different needs and preferences of older persons receiving palliative care (interRAI, n.d.).

The interRAI PC instrument was developed by the multinational consortium interRAI as part of the interRAI suite of instruments (interRAI Long-term Care facilities, interRAI Acute Care, interRAI Home Care, etc.) (interRAl.org). In Belgium, the interRAl suite of instruments are accessible through the online web application BelRAI (belrai.org). The interRAI PC instrument can be used in all healthcare settings to facilitate care planning and case mix and outcomes research (Steel et al., 2003). The interRAI PC instrument consists of 17 sections (see table 1) (Smith et al., 2010).

Outcomes of the interRAI PC instrument are Client Assessment Protocols (CAPs) and scales. The CAPs of the interRAI PC instrument are results that inform whether the client's condition still can improve or whether improvement potential is lacking. The goals of care of different CAPs vary and contain the possibility to solve problems, to reduce decline or to create an atmosphere of improvement. Every CAP is linked to guidelines on the 
online wiki manual which inform care providers about possible approaches to the problems. The scales of the interRAI PC instrument are coherent calculations of client characteristics. These scales are conform to internationally validated scales (e.g. Depression Rating Scale, Cognitive Performance Scale, etc.) (Dosa et al., 2006; Declercq et al., 2008; Declercq et al., 2009).

Research has shown the interRAI PC instrument to be a reliable instrument since 50 percent of the questions have a kappa value of .80 or higher (Steel et al., 2003; Hirdes et al., 2008). The interRAI PC instrument has been used in palliative home care research to evaluate determinants of death, determinants of Do-Not-Resuscitate Orders and the risk of developing pressure ulcers (Hirdes et al., 2008; Brink et al., 2008; Brink and Smith, 2008; Brink et al., 2006). Hirdes et al. (2012) have implemented the interRAI PC instrument to predict caregiver distress among palliative home care clients in Ontario (Hirdes et al., 2012). However, research on the experiences with the use of the interRAI PC instrument in nursing homes is scarce. The instrument has been used in several countries (e.g. Canada, France, Japan, etc.) (Hirdes et al., 2008) but no international studies have been conducted on the usefulness, feasibility and face validity of the interRAI PC instrument in residential care.

Therefore, this study aims to evaluate the (1) usefulness, (2) feasibility and (3) face validity of the interRAI PC instrument in nursing homes, based on experiences of care professionals of the nursing home staff. 
Table 1. Sections of the interRAI Palliative Care instrument
A. Identification information
B. Intake and initial history
C. Health conditions
D. Nutritional status
E. Skin condition
F. Cognition
G. Communication
H. Mood
I. Psychosocial well-being
J. Functional status
K. Continence
L. Medications
M. Treatments and procedures
N. Responsibility/Directives
O. Social supports
P. Discharge
Q. Assessment information 


\section{METHODS}

This study is part of a complex intervention to evaluate the effect of using the interRAI Palliative Care instrument on the quality of palliative care in nursing homes. The intervention consisted of 2 phases: 1) the preparatory phase; and 2) the implementation phase. A detailed description on the steps of the intervention can be found in the study protocol (Hermans et al. 2014).

\section{Study design}

A qualitative study was conducted, based on the abductive reasoning or inference approach. Abductive reasoning lies at the heart of 'grounded theorizing' (Coffey and Atkinson 1996). Abductive reasoning combines inductive and deductive reasoning. We start from the particular and try to relate the particular phenomenon to broader concepts and existing knowledge about other phenomena (Declercq 2000). As Coffey and Atkinson (1996) state, abductive reasoning seeks to go beyond the data by locating them into interpretative frameworks, but also includes new observations for coming up with new configurations of ideas. The consolidated criteria for reporting qualitative research (COREQ-32) were used in the description of the study (Tong et al. 2007).

\section{Participants and setting}

Calls for participation were sent out by four umbrella organizations of Flemish nursing homes (Belgium) and at a national conference for nursing home staff. Care professionals of 15 nursing homes voluntarily agreed to participate in the study. The goals of the study and the engagement requirements were explained during an introductory session in the participating nursing homes. During the next meeting, care professionals of the nursing homes received a training on the use of the interRAI Palliative Care instrument and the BeIRAI web application.

In the course of one year, these care professionals filled out the interRAI PC instrument every three months for all residents aged 65 years or older with palliative care needs. The need for palliative care was evaluated by means of the "surprise question" ('Would you be surprised if this person was to die within 6 to 12 months?') The answers to this question were discussed during multidisciplinary team meetings (MDT). Research showed that the 'surprise question' is a feasible, effective and simple screening tool to identify people with a significantly higher risk of mortality in the next year (Hubbard, 2011; Moss et al., 2008; Cohen et al., 2010).

\section{Data collection}

Multiple methods of qualitative data collection were used, combining notes of care professionals in participating nursing homes, semi-structured interviews and focus group discussions. Data were gathered between December 2013 and March 2015. 


\section{Notes}

Care professionals were asked to write down their experiences with the use of the interRAI PC instrument. These notes were used as a guideline during the interviews and the focus groups.

\section{Semi-structured interviews}

Care professionals of all 15 participating nursing homes were invited to take part in semi-structured interviews to gain perception of their experiences with the interRAI PC instrument. We started from existing ideas about the use of the instrument, which were based on knowledge retrieved from the BeIRAI project (Declercq et al., 2008; Declercq et al., 2009). General questions on the usefulness, feasibility and face validity were also included in the interview guide. In total, 19 care professionals of the multidisciplinary staff of the 15 participating nursing homes were interviewed by a researcher $(\mathrm{KH})$, assisted by a research student $(\mathrm{VC})$. The interviews took place in the nursing homes and lasted about one hour. Field notes were made during the interviews.

\section{Focus group discussions}

In addition to the semi-structured interviews, two focus groups were organized. Through a facilitated group discussion, people are more likely to provide responses (Mansell et al., 2004). Each focus group discussion was facilitated by a moderator $(\mathrm{KH})$ and lasted about two hours. Focus groups were based on three themes, guided by the results of the semi-structured interviews: (1) 'What are your experiences with the usefulness of the interRAI PC instrument?'; (2) 'What are your experiences with the feasibility of the interRAI PC instrument?'; (3) 'Is the content of the interRAI PC instrument accurate, clear and complete? Are there any missing, redundant or difficult items?'. In addition to the three main questions, care professionals were also asked about recommendations for good practices.

\section{Data analyses}

Notes taken by the care professionals were transcribed and anonymized. Interviews and focus groups were audio recorded and then transcribed verbatim. Two researchers compared and discussed the codes (KH, NS). Qualitative analyses were performed using NVivo version 10. Two researchers independently analyzed all results of the notes, interviews and focus groups $(\mathrm{KH}, \mathrm{NS})$. This analysis provides an overview of the usefulness, feasibility and face validity of the interRAI PC instrument as a comprehensive geriatric assessment. Results of the analysis were then mapped, based on positive aspects, negative aspects and practice based recommendations. The accuracy of the analysis was validated by six researchers independently (KH, VC, NS, JC, CVA, AD) and by the participants of the study. Table 2 shows an overview of all evaluation aspects. 
Table 2. Overview of all evaluation aspects and accompanying definitions

\begin{tabular}{ll}
\hline Evaluation aspects & Definitions \\
\hline Usefulnessa $^{a}$ & "The quality of being useful, to which extent something is useful." \\
Feasibilitya & "The convenience and suitability to use an instrument." \\
Face validity & "The degree to which an instrument appears to reflect the variable it has been designed \\
& to measure."
\end{tabular}

a(The American Heritage Dictionary of the English Language, 2009)

$\mathrm{b}$ (Research methods knowledge base, 2006)

\section{Rigour}

Several strategies were used to increase trustworthiness of the data and of the analyses. The study was based on the abductive reasoning or inference approach. An interview guide was developed by four researchers (KH, NS, CVA, AD) and was pilot tested during the BelRAI project (Declercq et al. 2008; Declercq et al. 2009). Interviews and focus groups were conducted by the principle author $(\mathrm{KH})$, who was assisted by a research student (VC). Two researchers compared and discussed the codes and independently analyzed all data (notes, interviews and focus groups) (KH, NS). Results of the analyses were mapped by two researchers $(\mathrm{KH}, \mathrm{NS})$. Data saturation was assumed since opinions and experiences of nursing home staff of all participating nursing homes were considered. The accuracy of the results and the analyses was validated by five researchers $(\mathrm{KH}, \mathrm{NS}, \mathrm{JC}, \mathrm{CVA}, \mathrm{AD})$ independently and by the participants of the study. Participants were asked to carefully read through the interview transcripts and the interpretation of the researchers in order validate the accuracy of the findings.

\section{Ethical considerations}

Approval to conduct this research was granted by the Belgian Commission for the Protection of Privacy (BCPP) and the UZ Leuven Medical Ethics Committee (file number B322201421986). Nursing home residents with palliative care needs or their representatives were asked to sign an informed consent agreement. Refusal did not affect the care provided to the resident. Residents or their representatives were able to withdraw their consent at any time. A formal procedure was undertaken to enable care professionals to complete the interRAI PC instrument on the secured web application. 


\section{RESULTS}

\section{Sample}

In the 15 participating nursing homes, 328 residents with palliative care needs were assessed during one year. In total, 19 care professionals participated in the interviews (Table 3). Five care professionals participated in the first focus group and 5 care professionals participated in the second focus group. Most participants were female $(n=$ 12) (Table 3). The average age of the healthcare workers was 47,2 years. Professional experience with the interRAI instruments ranged from 0 to 9 years.

Table 3. Characteristics of participants to the interviews and focus groups

\begin{tabular}{|c|c|c|c|c|c|c|}
\hline Participant & Profession & Gender & Age & $\begin{array}{l}\text { Experience } \\
\text { with the } \\
\text { interRAl } \\
\text { instruments }\end{array}$ & $\begin{array}{l}\text { Participation } \\
\text { to interviews }\end{array}$ & $\begin{array}{l}\text { Participation } \\
\text { to focus } \\
\text { groups }\end{array}$ \\
\hline 1 & Head nurse & $\mathrm{F}$ & 57 & Yes & $\mathrm{X}$ & $\mathrm{X}$ \\
\hline 2 & Head nurse & $\mathrm{F}$ & 44 & No & $x$ & $x$ \\
\hline 3 & $\begin{array}{l}\text { Quality } \\
\text { coordinator \& } \\
\text { physiotherapist }\end{array}$ & $M$ & 36 & No & $x$ & $x$ \\
\hline 4 & $\begin{array}{l}\text { Psychologist } \\
\text { and pastoral } \\
\text { worker }\end{array}$ & $\mathrm{F}$ & 38 & No & $x$ & \\
\hline 5 & Head nurse & $F$ & 42 & No & $x$ & $x$ \\
\hline 6 & Director & M & 64 & No & $x$ & $x$ \\
\hline 7 & Director & M & 43 & Yes & $x$ & \\
\hline 8 & Nurse & $\mathrm{F}$ & 40 & Yes & $x$ & $x$ \\
\hline 9 & Nurse & $\mathrm{F}$ & 52 & Yes & $x$ & \\
\hline 10 & Head nurse & M & 37 & Yes & $x$ & $x$ \\
\hline 11 & Head nurse & $M$ & 49 & Yes & $x$ & \\
\hline 12 & Physiotherapist & $\mathrm{F}$ & 56 & No & $x$ & $x$ \\
\hline 13 & $\begin{array}{l}\text { Quality } \\
\text { coordinator }\end{array}$ & $\mathrm{F}$ & 54 & No & $x$ & \\
\hline 14 & Nurse & $\mathrm{F}$ & 32 & No & $x$ & \\
\hline 15 & Nurse & $\mathrm{F}$ & 61 & No & $x$ & $x$ \\
\hline 16 & Physiotherapist & M & 61 & No & $x$ & \\
\hline 17 & Nurse & $\mathrm{F}$ & 37 & No & $x$ & $x$ \\
\hline 18 & Nurse & $\mathrm{F}$ & 49 & No & $x$ & \\
\hline 19 & Nurse & M & 45 & No & $x$ & \\
\hline
\end{tabular}




\section{Usefulness, feasibility and face validity of the interRAI Palliative Care instrument as a comprehensive geriatric assessment (table 4)}

\section{Usefulness of the interRAI PC instrument}

The usefulness of the interRAI PC instrument was explicitly mentioned as a positive aspect. Care professionals indicated that filling out the instrument improved the follow-up of residents with palliative care needs. Furthermore, the interRAI PC instrument was considered to be a suitable and detailed diagnostic and observation tool. The completion of the instrument also appeared to facilitate the dialogue with residents and their family. Additionally, the use of the interRAI PC instrument was believed to promote a common language and improved multidisciplinary teamwork.

Citation: "Filling out the interRAI PC instrument provides an added value during multidisciplinary team meetings. The content can also be discussed with the resident and his family."

Care professionals mentioned the standardized and objective collection of data as a strength of using the interRAI PC instrument. Although the confirmatory character of the results was pointed out as a positive aspect, one care professional also indicated that it was a drawback, since confirmatory results did not provide added value to improve care. Care professionals also indicated that the use of the interRAI PC instrument should be integrated in the daily care organization.

Citation: "I believe that it is important that the use of the interRAI PC instrument and the way in which the instrument is used, is part of the written policy of the organization."

Citation: "The project fails if it is not borne by the whole organization."

In order for the interRAI PC instrument to be used optimally, care professionals suggested that all healthcare settings should fill out the interRAI suite of instruments and collaborate to enable a transfer of client data between settings.

Furthermore, the fact that only eleven client assessment protocols (CAPs) (cf supra) were generated from the full instrument was evaluated as a limitation. The participants wanted more CAPs to be added, for example a CAP on the eyesight of the resident, a CAP on the sense of hearing of the resident, a CAP on psychosocial wellbeing, etc. Other suggestions for good practices concerned the fact that Resource Utilization Groups (RUGs) should be added as outcomes of the interRAI PC instrument. RUGs is a case-mix index for resource utilization for a nursing home resident (Fries et al., 1994; Dellefield, 2006). Care professionals also recommended to add Quality Indicators (Qls) as an outcome of the interRAI PC instrument, since these can be used to make comparisons between organizations. Furthermore, they can be used to stimulate improvements in healthcare delivery services (Hibbert et al., 2013). 


\section{Feasibility of the interRAI PC instrument}

Care professionals who already had experience with filling out the interRAI Long-Term Care Facilities instrument indicated that it was more feasible to fill out the interRAI PC instrument for people with palliative care needs, since the interRAI PC instrument is less extensive. Another strength was the fact that the completion time evolved. The first assessments approximately took 45 minutes to complete. After one year, filling out the interRAI PC instrument approximately took 20 minutes. One of the most important drawbacks was that filling out the instrument was an extensive, laborious and time consuming process.

Citation: "Because of the large quantity, filling out the interRAI PC instrument is a very slow process. There is also other work that needs to be done"

Under a heavy workload, there was also not always sufficient time for care professionals to fill out the instrument.

Citation: "I often completed the interRAI PC instrument individually because my colleagues didn't have enough time while burdened with other tasks."

Furthermore, for many items, the last three days have to be taken into consideration. This was experienced as not convenient since care professionals often have not been working during all three of the last days. Another negative aspect was that guidelines were not always practice based, according to the respondents.

Preconditions for using the interRAI PC instrument, such as the necessary availability of computers in nursing homes, the fact that planning and organization was required and the informed consent requirements resulted in completion delays.

Citation: "There were a lot of delays because the informed consents had to be signed by family members. Therefore, the work is not being done. You have to see the family, you have to be in the nursing home and you have to think about the informed consent. It takes a while until you have all the consents."

Furthermore, training packages were available for care professionals of healthcare settings that participated in a study project. A number of care professionals received a training on the interRAI suite of instruments and the BeIRAI web application and relayed this information back to other care professionals of their healthcare settings. However, no general yearly trainings are available. Care professionals asked for the organization of yearly trainings concerning the interRAI suite of instruments and the BeIRAI web application. Funding is also needed to coordinate the integration of the instrument in the organization and to reduce workload. Furthermore, since it is currently not obligatory to fill out the interRAI PC instrument, care professionals experienced the completion of the instrument as extra work on top of mandatory tasks instead of experiencing it as an added value.

Citation: "The fact that it is currently not mandatory to fill out the interRAI PC instrument is a barrier for us to fill out the instrument. That is in addition to other work."

The BelRAI web application as a tool to fill out the interRAI PC instrument was evaluated as a secured web application that stimulates multidisciplinarity and enables a transfer of patient data between settings. However, the user-friendliness of the BeIRAI system requires optimization since privacy settings are very strict, the login 
procedure is cumbersome, there is no link with the electronic patient file, etc. Care professionals recommended to optimize the usability of the BeIRAI web application in order to complete the interRAI PC instrument.

Finally, care professionals indicated that the multidisciplinary completion of the instrument should be mandatory. Some care professionals filled out a complete interRAI PC instrument on their own. This made the completion laborious and time-consuming and prevented multidisciplinary and transdisciplinarity.

\section{Face validity of the interRAI PC instrument}

As to the face validity of the interRAI PC instrument, all care professionals stated that the results provided a clear and overall picture of the residents with palliative care needs. In most cases, results were adequate and confirmatory. Care professionals also indicated that the CAPs were good indicators of what they were supposed to measure. Furthermore, filling out the interRAI PC instrument led to more attention for spirituality (section I. Psychosocial Well-being).

A face validity problem of the instrument included that the fact the interRAI PC instrument is not setting-specific and that some items are only relevant to the home care setting and some only to the acute care setting. These items were difficult to fill out in a nursing home setting.

Citation: 'In section $M$ 'Treatments and procedures', the items home nurse, home health aides, homemaking services, meals, etc. refer to home care. I would leave these items out in the nursing homecare setting."

Additionally, several items were perceived as difficult to fill out for people with dementia. Evaluating psychosocial wellbeing (Section I. Psychosocial Well-being) and the intensity of pain (Section C. Health conditions) was then based on the care professional's interpretation and estimation. Some items were perceived as unclear. Items on psychosocial wellbeing and the intensity of pain were for instance difficult to complete since several nursing homes were not able to express themselves because they suffered from cognitive impairment of dementia. Therefore, answers were often based on the personal interpretation of the care professionals. Certain items on psychosocial wellbeing were also judged as difficult to understand: 'Does the person have inner strengths that can be enforced?', 'Does the person find meaning in day-to-day life?', 'Is the person at peace with life?'. Furthermore, certain items were considered to be redundant. The item of travelled distance in a wheelchair (Section J. Functional status) was evaluated as uninformative. The medication section was considered to be redundant as it overlapped with information already recorded in the electronic patient file.

Several sections and items were judged as incomplete. Items regarding mouth care (Section D. Nutritional status) were judged as not comprehensive enough.

Citation: "Section $K$ regarding mouth care is not comprehensive enough. It only asks information on whether the person has a dry mouth. However, when a person has palliative care needs, there are much 
more mouth problems. We use the Oral Health Assessment which concerns lips, tongue, teeth, dentures, etc."

The item toilet transfer (Section J. Functional status) was critiqued for not having a possibility to click an option that the activity did not occur. Additionally, the lacking of items on comfort care, the feelings of the resident, palliative sedation, euthanasia and the involvement of a clerical worker was mentioned as a limitation of the instrument.

Care professionals argued for more suitable items for people with dementia. These items should be based on observation.

Citation: "We use extra questionnaires for people with dementia. For example the Pain Scale... This scale can be scored by means of an observation of the person's mimicry. It would be interesting if certain scales would be integrated in the interRAI PC instrument."

Finally, certain items (especially items on psychosocial wellbeing) should be simplified according to the respondents.

Table 4 shows a general overview of the results. 
Table 4. Overview of the usefulness, feasibility and face validity of the interRAI Palliative Care instrument, subdivided on the basis of positive aspects, negative aspects and practice based recommendations.

\begin{tabular}{|c|c|c|c|}
\hline Evaluation aspects & Positive aspects & Negative aspects & Practice based recommendations \\
\hline Usefulness & $\begin{array}{l}\text { - Better follow-up of residents with } \\
\text { palliative care needs } \\
\text { - Observation tool } \\
\text { - Diagnostic tool } \\
\text { - Facilitates dialogue with the residents } \\
\text { and their family } \\
\text { - Promotes multidisciplinarity } \\
\text { - Data-collection is standardized and } \\
\text { neutral }\end{array}$ & $\begin{array}{l}\text { - Results are often confirmatory, } \\
\text { without adding new information } \\
\text { - Lacking CAPs: vision, psychosocial } \\
\text { wellbeing and constipation }\end{array}$ & $\begin{array}{l}\text { - Integrate the use of the interRAI PC } \\
\text { instrument in the organization of daily } \\
\text { care routines } \\
\text { - Promote and support the } \\
\text { multidisciplinary completion of the } \\
\text { interRAI PC instrument } \\
\text { - Use the interRAI PC instrument in } \\
\text { acute care, home care and residential } \\
\text { care settings to enable a transfer of } \\
\text { client data between settings } \\
\text { - Provide more practice based } \\
\text { guidelines } \\
\text { - Add RUGS } \\
\text { - Add quality indicators } \\
\text { - Add more CAPs (e.g. psychosocial } \\
\text { wellbeing, vision, hearing, etc.) }\end{array}$ \\
\hline Feasibility & $\begin{array}{l}\text { - Less extensive than the interRAI Long- } \\
\text { term Care Facilities instrument } \\
\text { (nursing home version) } \\
\text { - Completion time evolves (less time is } \\
\text { needed when used more frequently) } \\
\text { - Multidisciplinary completion } \\
\text { - Secured BelRAI web application that } \\
\text { allows a transfer of data from one } \\
\text { assessment to the next assessment }\end{array}$ & 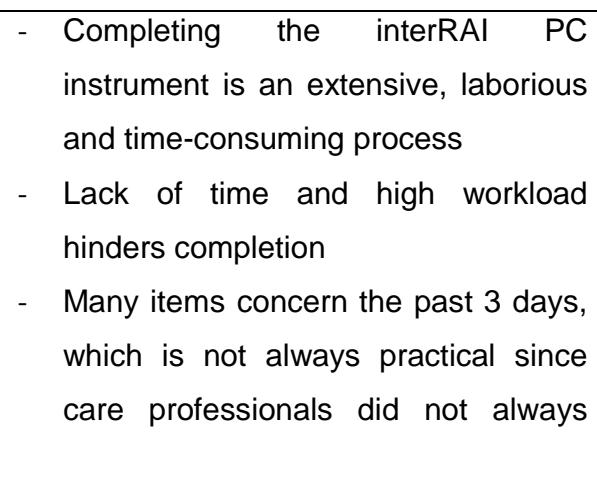 & 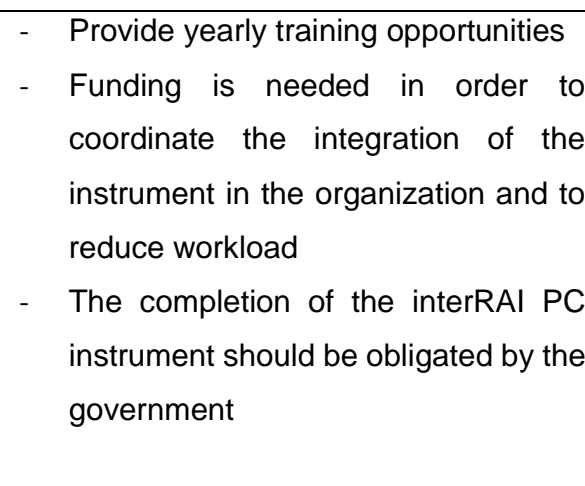 \\
\hline
\end{tabular}


have contact with the residents every

day

- Guidelines are not always customized to the individual situation of the person

- Guidelines are not always perceived as practice-based

- There are not enough computers available in the nursing homes

- Planning and organization in the nursing home setting is required

- Informed consent results in completion delays

- A complete assessment was not always possible due to the high workload, the turnover of staff and absenteeism

- User-friendliness of the BelRAI web application (login problems, strict privacy settings, no integration with electronic patient file, etc.)

- More attention is paid to spirituality

- The CAPs are good indicators of what they are supposed to measure (Mood, Pain, Nutrition, etc.)
Optimize the user-friendliness of BelRAl (login procedure, roles, length, lay-out, tablet version, etc.)
- Not setting-specific: some items concern home care, acute care and residential care settings

Several items are difficult to complete for residents with dementia: psychosocial wellbeing, intensity of pain, activities of daily living and advance directives
More fitting items for residents with dementia (e.g. items based on observation)

- Make the instrument setting-specific - Complete incomplete items

- Clarify difficult items (especially items on psychosocial wellbeing) $(F)$ 
- Unclear, complex items: items on psychosocial wellbeing and pain intensity

- Redundant items: travelled distance in wheelchair does not say much and the medication section overlaps with electronic patient file

- Incomplete items: mouth care (oral assessment is needed on lips, tongue, teeth, dentures, etc.), toilet transfer and advance directives

- Lacking items: comfort care, feelings of the resident, palliative sedation, euthanasia, involvement of clerical

worker

interRAI Palliative Care instrument = interRAI PC instrument; Client Assessment Protocols = CAPs; Resource utilization Groups = RUGs. 


\section{DISCUSSION}

In general, the interRAI Palliative Care (interRAI PC instrument) is a useful instrument according to care professionals in nursing homes. However, it is not always feasible to complete the interRAI PC instrument because of organizational reasons. Furthermore, the face validity of the instrument could be improved since certain items are incomplete, lacking, redundant or too complex.

Based on interviews and focus groups with care professionals of 15 nursing homes, primary positive aspects of the interRAI PC instrument are considered to be the fact that its completion enables a better follow-up of residents with palliative care needs. Furthermore, it is an observation tool that provides an overall picture of the nursing home residents. Main negative aspects include the fact that filling out the interRAI PC instrument is an extensive and timeconsuming process. Under a heavy workload, care professionals may not always have sufficient time to complete the instrument (Carayon and Gurses, 2008). Management should support and stimulate the multidisciplinary completion of the instrument. Time to fill out the interRAI PC instrument should therefore be incorporated in the job description of care professionals. Many care professionals also indicate that informed consent requirements result in delays to complete the interRAI PC instrument. However, in nursing homes where the use of the interRAI instruments was already integrated, informed consents were signed during the intake procedure and therefore did not cause extra delays. The use of the instrument should thus be integrated in the organization of daily care routines. A remarkable finding was also that care professionals who had experience with the completion of the interRAI instruments, listed more positive aspects of the interRAI PC instrument than did care professionals who did not have any experience before the project started. According to these care professionals, it would take at least one year to implement the use of the interRAI PC instrument and the web application in the daily routines of the organization. Probably, from then onwards, one can begin to experience the benefits of using the interRAl instruments and the subsequent outcomes. Furthermore, the BelRAl system is described as a secured web application that allows multidisciplinary completion of the interRAI instruments and that enables a transfer of data between settings. However, the user-friendliness of the BeIRAI web application requires optimization. These findings confirm results of the studies of Devriendt et al. (2013) and Vanneste and Declercq (2014), stating that the BelRAI software provides an optimal security protection. However, weaknesses include the user-friendliness of particular features and the complex architecture of the BeIRAI software due to privacy regulations. Future research should evaluate the positive and negative aspects of the interRAI PC instrument and the BelRAI web application after an implementation period of more than one year.

Care professionals stated that several items of the interRAI PC instrument are not specifically focusing on the nursing home setting but concern the home care or acute care setting. This makes the instrument very extensive and sometimes even confusing for care professionals to complete. These findings suggest that the interRAI PC 
instrument should be made more setting-specific. However, an optimal balance between setting-specific and shared items should be guaranteed since the instrument aims to generate transferable data.

Additionally, the section 'Psychosocial Wellbeing' is difficult to complete according to the care professionals. This section contains items about psychosocial wellbeing and spirituality. Although psychosocial wellbeing and spirituality are of particular importance in older adults at the end of life (Wallace and 0'Shea, 2007; Narayanasamy et al., 2004), spirituality in older adults is often neglected (Wallace and 0'Shea, 2007). This study shows that by using the interRAI PC instrument, more attention is drawn to psychosocial wellbeing and spirituality since the instrument contains several items on this topic. However, care professionals mention that answers on these psychosocial and spiritual items in the interRAI PC instrument are often based on the personal observation and interpretation of the care professionals. The items are therefore difficult to complete, especially for people in a later stage of dementia who are not always able to express themselves. This can lead to bias since certain factors might be over- or underreported, based on the personal perception of the care professionals. Secondly, the formulation of certain questions is too complex (e.g. 'Does the person have inner strengths that are enforced?'). Finally, results on psychosocial wellbeing and spirituality should be reflected in the outcomes of the interRAI PC instrument. For the above reasons, the section 'Psychosocial Wellbeing' of the interRAI PC instrument should contain clear and validated items concerning psychosocial and spiritual needs. It would be recommended to replace the current section on Psychosocial Wellbeing of the interRAI PC instrument by an international validated scale that assesses psychosocial and spiritual needs for people with and without dementia (e.g. Palliative Care Needs Assessment) and to add CAPs concerning psychosocial and spiritual needs.

As mentioned before, care professionals indicate that it is very difficult to complete the interRAI PC instrument for residents with dementia since answers on several items (pain, psychosocial wellbeing, etc.) are often based on the personal interpretation of the care professionals. An adapted interRAI PC instrument should be developed for people with dementia, containing items that are more based on observation. Items about pain could for instance be replaced by the Pain Assessment in Advanced Dementia (PAINAD) (Warden, Hurley, Volicer, 2003).

Several items of the interRAI PC instrument are evaluated by care professionals as redundant, incomplete or even lacking. One example concerns items on palliative sedation and euthanasia. In Belgium, euthanasia has been legalized since September 2002 (Before you go, 2002). The interRAI PC instrument should be adapted to countryspecific laws and to changes in laws and policies.

\section{Limitations}

Limitations of the study need to be acknowledged. Firstly, care professionals of 15 nursing homes participated in the study. This sample might not be representative for the overall Belgian nursing home population. Furthermore, the interRAI PC instrument was only filled out during the period of one year. This might be a limitation of this study 
since care professionals indicate that it approximately takes one year to integrate the use of the interRAI instruments and the BelRAI web application in a nursing home. Because of the time restrictions, it was not possible for all care professionals to use the outcomes of the instrument for the evaluation, adaptation or development of care plans within the course of the project. Future research should therefore focus on the barriers and facilitators of using the outcomes of the interRAI PC instrument in order to evaluate, adapt and develop care plans.

\section{Conclusion}

This is the first study to evaluate the usefulness, feasibility and face validity of the interRAI Palliative Care instrument (interRAI PC instrument) in nursing homes, based on an abductive reasoning approach. It found the interRAI PC instrument to be a useful instrument according to care professionals in nursing homes, but also identified issues with completion of the instrument because of organizational reasons (e.g. high workload, lack of computers, lack of management support, etc.) and identified a need to improve the face validity of the instrument with certain items indicated as incomplete, lacking, redundant or too complex.

Our results incite the multinational consortium interRAI to optimize the interRAI PC instrument, especially with respect to the content of the instrument. Our study also indicates that, in order to fully exploit the results of the interRAI PC instrument, it should be integrated in the organization of daily care routines in the nursing homes. Management should support and stimulate the completion of the instrument. Time to fill out the interRAI PC instrument should be incorporated in the job description of care professionals.

Tackling the critical remarks of care professionals will help to optimize the interRAI Palliative Care instrument and hence support palliative care of high quality in nursing homes.

\section{Acknowledgements}

The study is part of 'Flanders Study to Improve End-of-life Care and Evaluation Tools' (FLIECE), a collaboration between VUB, UGent, KU Leuven and VUmc Amsterdam. This project is funded by the Flemish government Agency for Innovation by Science and Technology (SBO IWT nr. 100036).

The authors would like to thank Valerie Castermans (VC), a master's student 'Social Work and Policy' who worked under the supervision of $\mathrm{AD}$ and $\mathrm{KH}$, for her contribution to the data collection and the analyses.

\section{Conflict of interest}

We declare that we have no competing interests. 


\section{REFERENCES}

Before you go, 2002. Euthanasiewet België. Available at: http://www.beforeyougo.be/nl/weesvoorbereid/euthanasie/euthanasiewet-belgie?gclid=CliW656W2MYCFcXHtAodBdgKSg (accessed 15.12.2015) .

Bernabei, R., Venturiero, V., Tarsitani, P., Gambassi, G, 2000. The comprehensive geriatric assessment: when, where, how. Crit Rev Oncol Hematol. 33, 45-56. doi 10.1016/S1040-8428(99)00048-7

Brink, P., Smith, T.F., 2008. Determinants of home death in palliative home care: using the interRAI Palliative Care to Assess End-of-Life Care. Am J Hosp Palliat Med. 25(4), 263-70. doi: 10.1177/1049909108319261

Brink, P., Smith, T.F., Kitson, M., 2008. Determinants of Do-not-Resuscitate Orders in palliative home care. J Palliat Med. 11, 226-232. doi: 10.1089/jpm.2007.0105

Brink, P., Smith, T.F., Linkewich, B., 2006. Risk of developing pressure ulcers among palliative home care clients. J Palliat Med. 11, 226-232. doi:10.1089/jpm.2006.9.1369

Carayon, P., Gurses, A.P., 2008. Nursing workload and patient safety - A human factors engineering perspective, in Hughes, R.G. (Ed), Patient safety and quality: An evidence based handbook for nurses. Agency for Healthcare Research and Quality, Rockville, MD.

Christopher, M., 2000. Benchmarks to improve end-of-life care. Midwest Bioethics Center, Kansas City, Missouri.

Coffey, A., Atkinson, P., 1996. Making sense of qualitative data. Complementary research strategies. Sage, Thousand Oaks.

Cohen, L.M., Ruthazer, R., Moss, A.H., Germain, M.J., 2010. Predicting six-month mortality for patients who are on maintenance hemodialysis. Clin J Am Soc Nephrol. 5, 72-79. doi 10.2215/CJN.03860609.

Davies, E., Higginson I.J., 2004. Better palliative care for older people. WHO regional office for Europe, Copenhagen, Denmark.

Declercq, A., 2000. De complexe zoektocht tussen orde en chaos: een sociologische studie naar de differentiatie in de institutionele zorgregimes voor dementerende ouderen. Departement Sociologie, KU Leuven, Leuven.

Declercq, A., Gosset, C., Paepen, B., de Almeida Mello, J., Vanneste, D., Detroyer, E., et al., 2008. Actieproject BelRAI II: Haalbaarheid van de RAl-methode in België. Eindrapport in opdracht van het Ministerie van Volksgezondheid, Veiligheid van de Voedselketen en Leefmilieu, Brussel, België. 
Declercq, A., Gosset, C., Paepen, B., de Almeida Mello, J., Vanneste, D., Detroyer, E., et al., 2009. Eindrapport in opdracht van het Ministerie van Volksgezondheid, Veiligheid van de Voedselketen en Leefmilieu, Brussel, België.

Dellefield, M.E., 2006. Using the Resource Utilization Groups (RUG-III) System as a Staffing Tool in Nursing Homes. Geriatr Nurs. 27(3), 160-165. doi 10.1016/j.gerinurse.2006.02.004

Devriendt, E., Wellens, N., Flamaing, J., Declercq, A., Moons, P., Boonen, S., Milisen, K., 2013. The interRAI Acute Care instrument incorporated in an eHealth system for standardized and web-based geriatric assessment: strengths, weaknesses, opportunities and threats in the acute hospital setting. BMC Geriatrics. $13,90$. doi:10.1186/1471-2318-13-90

Dosa, D., Bowers, B., Gifford, D.R., 2006. Critical review of resident assessment protocols. J Am Geriatr Soc. 54(4), 659-66. doi: 10.1111/j.1532-5415.2006.00654.x.

Fries, B.E., Schneider, D.P., Foley, W.J., Gavazzi, M., Burke, R., Cornelius, E., 1994. Refining a Casemix Measure for Nursing Homes: Resource Utilization Groups (RUG-III). Med Care. 32(7), 668-685.

Froggatt, K., Payne, S., 2006. A survey of end-of-life care in care in care homes: issues of definition and practice. Health Soc Care Community. 14(4), 341-348. doi: 10.1111/j.1365-2524.2006.00628.x

Hermans, K., de Almeida Mello, J., Spruytte, N., Cohen, J., Van Audenhove, Ch., Declercq, A., 2014. A comparative analysis of comprehensive geriatric assessments for use in nursing homes: a systematic review. J Am Med Dir Assoc. 15(7), 467-76. doi: 10.1016/j.jamda.2014.01.002

Hermans, K., Spruytte, N., Cohen, J., Van Audenhove, Ch., Declercq, A., 2014. Informed palliative care in nursing homes through the interRAI Palliative Care instrument: a study protocol based on the medical research council framework. BMC Geriatrics. 14, 132. doi:10.1186/1471-2318-14-132, 132

Hibbert, P., Hannaford, N., Long, J., Plumb, J., Braithwaite, J., 2013. Final Report: Performance indicators used internationally to report publicly on healthcare organisations and local health systems. Australian Institute of Health Innovation, University of New South Wales.

Hirdes, J.P., Freeman, S., Smith, T.F., Stolee, P., 2012. Predictors of caregiver distress among palliative home care client in Ontario: Evidence based on the interRAI Palliative Care (interRAI PC instrument). Palliat Support Care. 10, 155-163. doi: $10.1017 /$ S1478951511000824 
Hirdes, J.P., Ljunggren, G., Morris, J.N., Frijters, D.H., Finne Soveri, H., Gray, L., et al., 2008. Reliability of the interRAI suite of assessment instruments: a 12-country study of an integrated health information system. BMC Health Serv Res. 30(8), 277. doi:10.1186/1472-6963-8-277

Houttekier, D., Cohen, J., Surkyn, J., Deliens, L., 2011. Study of recent and future trends in place of death in Belgium using death certificate data: a shift from hospitals to care homes. BMC Public Health. 11, 228. doi: 10.1186/14712458-11-228

Hubbard, G., 2011. The 'surprise question' in end-of-life care. Br J Community Nurs. 16(3):109.

InterRAI. (n.d.). Palliative Care (PC). Available at: http://www.interrai.org/palliative-care.html (accessed 30.10.2015).

Kales, H.C., Gitlin, L.N., Lyketsos, C.G., 2015. Assessment and management of behavioral and psychological symptoms of dementia. Br Med J (clinical research ed.). 350, h369. doi:10.1136/bmj.h369.

Mansell I, Bennett G, Northway R, Mead, D., Moseley, L., 2004. The learning curve: the advantages and disadvantages in the use of focus groups as a method of data collection. Nurs Res. 11(4), 79-88. doi 10.7748/nr2004.07.11.4.79.c6217

Mcllfatrick, S., 2007. Assessing palliative care needs: views of patients, informal carers and healthcare professionals. J Adv Nurs. 57(1), 77-86. doi 0.1111/j.1365-2648.2006.04062.x.

Moss, A.H., Ganjoo, J., Sharma, S., Gansor, J., Senft, S., Weaner, B., et al., 2008. Utility of the "surprise" question to identify dialysis patients with high mortality. Clin J Am Soc Nephrol. 3, 1379-1384. doi: 10.2215/CJN.00940208

Narayanasamy, A., Clissett, P., Parumal, L., Thompson, D., Annasamy, S., Edge R., 2004. Responses to the spiritual needs of older people. J Adv Nurs. 48(1), 6-16. doi 10.1111/j.1365-2648.2004.03163.x

Osterweil, D., Brummel-Smith, K., \& Beck, J.C. (eds), 2000. Comprehensive Geriatric Assessment. McGraw Hill, New York, NY.

Percival, J., Johnson, M., 2013. End-of-life care in nursing and care homes. Nurs Times. 109(1-2), 20-22.

Phillips, J., Davidson, P.M., Jackson, D., Kristjanson, L., Daly, J., Curran, J., 2006. Residential aged care: the last frontier for palliative care. J Adv Nurs. 55(4), 416-24). doi 10.1111/j.1365-2648.2006.03945.x.

Research methods knowledge base, 2006. Available at: http://www.socialresearchmethods.net/kb/measval.php (accessed 24.11.2015) 
Rubenstein, L.Z., 1995. An overview of comprehensive geriatric assessment: rationale, history, program models, basic components, in: Rubenstein, L.Z., Wieland, D., Bernabei, R. (Eds), Geriatric Assessment Technology: The State of the Art. Springer, New York, NY.

Scanlan, B.C., 2005. The value of comprehensive geriatric assessment. Care Manag J. 6(1), 2-8.

Smith, T.F., Steel, K., Fries, B.E., Morris, J.N., Belleville-Taylor, P., Curtin-Telegdi, N., et al., 2010. interRAI Palliative Care (PC) Assessment Form and User's Manual. InterRAI, Washington, DC.

Steel, K., Ljunggren, G., Topinkova, E., Morris, J.N., Vitale, C., Parzuchowski, J., et al., 2003. The RAI-PC: An assessment instrument for palliative care in all settings. Am J Hosp Palliat Med. 20(3), 211-9.

Tong, A., Sainsbury, P., Craig, J., 2007. Consolidated criteria for reporting qualitative research (COREQ): a 32-item checklist for interviews and focus groups. Int J Qual Health Care. 19 (6), 349-357. http://dx.doi.org/10.1093/intqhc/mzm042

The American Heritage Dictionary of the English Language, 2009. Houghton Mifflin Harcourt, Boston, Massachusetts.

Unwin, B.K., Porvaznik, M., Spoelhof, G.D., 2010. Nursing home care: part I. Principles and pitfalls of practice. Am Fam Physician. 18(10), 1219-1227.

Vanneste, D., Declercq, A., 2014. The development of BelRAI, a web application for sharing assessment data on frail older people in home care, nursing homes and hospitals, in: Meyer, I., Müller, S., Kubitschke, L. (Eds). Achieving effective integrated E-care beyond the silos. IGI Global, Hershey, PA.

Wallace, M., 0'Shea, E., 2007. Perceptions of Spirituality and Spiritual Care Among Older Nursing Home Residents at the End of Life. Holist Nurs Pract. 21(6), 285-289. doi 10.1097/01.HNP.0000298611.02352.46

Warden, V., Hurley, A.C., Volicer, V. 2003. Development and psychometric evaluation of the Pain Assessment in Advanced Dementia (PAINAD) Scale. J Am Med Dir Assoc. 4, 9-15. Doi 10.1016/S1525-8610(04)70258-3.

Wieland, D., Hirth, V., 2003. Comprehensive Geriatric assessment. Cancer Control. 10(6), 454-462.

World Health Organization, 2002. WHO definition of palliative care. Available at: http://www.who.int/cancer/palliative/definition/en/ (accessed 09.09.2015). 
Wowchuck, S.M., McClement, S., Bond, J.Jr., 2007. The challenge of providing palliative in the nursing home part II: internal factors. Int J Palliat Nurs. 13(7), 345-50. doi 10.12968/ijpn.2007.13.7.24346. 